\title{
Automated extraction of treatment patterns from social media posts: an exploratory analysis in renal cell carcinoma
}

\author{
Sreeram V Ramagopalan*,1, Bill Malcolm², Evie Merinopoulou³, Laura McDonald ${ }^{1}$ \& \\ Andrew $\mathrm{Cox}^{3}$ \\ ${ }^{1}$ Centre for Observational Research \& Data Sciences, Bristol-Myers Squibb, Uxbridge UB8 1DH, UK \\ ${ }^{2}$ Worldwide Health Economics \& Outcomes Research, Bristol-Myers Squibb, Uxbridge UB8 1DH, UK \\ ${ }^{3}$ Real-World Evidence, Evidera, London W6 8DL, UK \\ *Author for correspondence: Tel.: +44 1895 523000; sreeram.ramagopalan@bms.com
}

\begin{abstract}
Aim: The use of health-related social media forums by patients is increasing and the size of these forums creates a rich record of patient opinions and experiences, including treatment histories. This study aimed to understand the possibility of extracting treatment patterns in an automated manner for patients with renal cell carcinoma, using natural language processing, rule-based decisions, and machine learning. Patients \& methods: Obtained results were compared with those from published observational studies. Results: 42 comparisons across seven therapies, three lines of treatment, and two-time periods were made; 37 of the social media estimates fell within the variation seen across the published studies. Conclusion: This exploratory work shows that estimating treatment patterns from social media is possible and generates results within the variation seen in published studies, although further development and validation of the approach is needed.
\end{abstract}

First draft submitted: 15 July 2019; Accepted for publication: 15 August 2019; Published online: 4 September 2019

Keywords: natural language processing • oncology $\bullet$ social-media $\bullet$ treatment patterns

A PwC Health Research Institute social media consumer survey found that $42 \%$ of participants use social media to learn about a healthcare issue, $29 \%$ sought information related to other patients' experiences with their disease, $25 \%$ had discussed a health-related experience and $20 \%$ had joined a health community or forum [1]. There is growing evidence that patients value social media forums for advice and discussion of their conditions [2-6]. While the 'social media' includes large, generic platforms such as Twitter, Facebook and Instagram, patients also use condition-specific discussion forums such as Cancer Survivors Network [7] and Diabetes Daily [8]. These sites contain posts from tens of thousands of patients and stretch back several years, creating a rich and valuable record of patient opinions and experiences. There is an increasing interest by the healthcare industry in the use of social media for research into understanding the patient perspective. Social media has been used to assess patient perspective in qualitative studies and safety signal detection, and as a monitor for adverse drug reactions in safety applications [9]. In June 2018, the US FDA published a draft guidance that encouraged stakeholders to explore the use of social media when conducting studies, particularly to gain insight into patients' perspectives regarding symptoms and impacts of their disease on overall quality of life. According to the FDA guidance, targeted social media research could be used to complement literature review findings, inform the development of research tools or serve as a supplement to traditional research approaches, including the development of tools such as patient questionnaires [10].

When considering a new data source such as social media, the question of potential bias naturally arises; however, it is important to remember that no study is free from any bias. The potential biases in social media are not yet well characterized, so it is important that future studies aim to establish the validity of information obtained from social media sources and to explore the potential for bias. The objective of this pilot study was to understand if it is possible to extract meaningful treatment history data in an automated manner for patients with renal cell carcinoma (RCC), using a combination of natural language processing, rule-based decisions (if-then-else) and machine learning. This exploratory study compared estimates derived from social media with those produced from published studies that

Future 8 Medicine 
utilized traditional approaches such as retrospective chart reviews to evaluate the validity of estimates for treatment proportions obtained from social media.

\section{Patients \& methods}

Social media data sources \& sample selection

Patient post data were extracted from the following social media websites: CancerCompass [11], Cancer Survivors Network [7], DailyStrength [12] and Macmillan Cancer Support [13]. These are publicly available websites that include discussion forums where patients and caregivers share information and receive support. In this study, data were extracted from discussions specific to RCC.

The likely geographical applicability of the social media websites was determined through Alexa Page Rank services [14], which detail the proportions of countries using the sites based on user IP address locations. Here, we assumed that the data from Alexa were consistent with the geographical locations of people posting on the sites. We retrieved information only from countries where English is spoken as the primary, or one of the primary languages (UK, Ireland, USA, Australia, New Zealand and Canada), as the social media data were restricted to the English language, but we reported the percentages from all countries.

Posts from metastatic RCC patients only were extracted through a machine learning algorithm trained on features of the posts' content, using a random sample of posts annotated manually as a training dataset; this gradient boost method identified metastatic users with an overall accuracy of $99.9 \%$. The quality of this process was checked through a manual review of a subsample of 100 randomly selected users predicted to be metastatic. Prediction of metastatic status was largely based on self-reporting stage of disease.

\section{Treatment pattern definition}

The study focused on evaluating treatment patterns for targeted therapies. Line of therapy was defined as the order in which the targeted therapies of interest to this study were administered, while other treatments were ignored and not counted as a line of therapy. The targeted therapies included were sunitinib, sorafenib, pazopanib, everolimus, temsirolimus, bevacizumab and axitinib. The listed treatments were chosen for the frequency with which they were reported and because they were the focus of previously published studies. Similarly, the time periods selected for the study were chosen to match the time periods of the published studies.

\section{Cleaning \& standardizing of text}

Text data were standardized and cleaned by the removal of nonstandard characters and symbols, and converted to the Unicode UTF-8 standard [15]. Spell correction was carried out using the Hunspell package [16] through the $\mathrm{R}$ statistical programming language. For lexical diversity, the seven target treatments were standardized using a custom lexicon, which contained all variations of treatment names obtained through using generalized Levenshtein edit distance [17]. Recognition of treatments, phrase negation (polarity), questions, and conditional and uncertain statements were carried out through the Apache cTakes Platform [18].

The text for each user was split into phrases, and phrases not containing target treatments were removed. The tense (past, present or future) of the phrases was identified using the Apache cTakes framework. Phrases referring to treatments where the phrase was negated, conditional or formed as a question were removed. Phrases referring to events in the future tense were removed. The remaining phrases were sorted based on posting date, starting from the earliest to the most recent. For phrases that mentioned the same treatment in temporally consecutive phrases, the later phrase was removed (on the assumption that in the treatment of RCC, it would be extremely rare for a patient to receive the same treatment twice in separate lines of therapy). Any duplicated phrases (often from cross-posting) were removed. At this stage, all words except for the seven target treatments were removed, leaving a sequence of nonrepeating treatment mentions which were not negated or conditional, did not form parts of questions and originated only within phrases in the past or present tense. However, links to the full text of the origin phrases were maintained for later checking and processing. The resulting sequential order of treatments was deemed to be the 'baseline' order in which the patient received treatments. This assumption was validated by manual review and reconstruction of treatment sequences from a sample of posts from 100 users. Overall, $78 \%$ of users reported treatments in chronological order. By searching and cross-referencing, original phrases containing temporal references ('in June...', 'five weeks ago...') were reviewed manually to determine if changes in the current baseline sequence of treatments were deemed necessary. If changes were deemed necessary, they were made manually. Determination of the treatments at line of therapy and the proportion of treatments by line was 
made from this data. As a form of quality control, a sample of the extracted treatment sequences was selected for comparison by manual review of the patients' post history.

\section{Comparisons with published studies}

The obtained results were compared with estimated proportions for the same targeted therapies that were acquired from published studies identified through a targeted literature review. A search was conducted via PubMed using the following search string: 'renal cell carcinoma' (all fields) AND ('secondary' [subheading] OR 'secondary' [all fields] OR 'metastatic' [all fields]) AND 'treatment patterns'(all fields).

Since the social media study and published studies were of different designs, the approach was to select studies that are as similar as possible. We included only studies that contained at least 200 patients with metastatic RCC and covered at least six of the seven treatments of interest. Included studies needed to be based in USA or the European Union Five (France, Germany, Italy, Spain and the UK), and to have taken place within the years 2003 through 2018 (Figure 1).

Two separate comparisons were made for the periods of 2004-2015 and 2009-2015 because of the many changes in treatment recommendations and new treatments becoming available.

\section{Targeted literature review}

Although 95\% CIs of the treatment proportion estimates were not given in publications, the exact binomial 95\% CIs were calculated. In some cases, the treatment proportions were not directly available from the publication but could be calculated from the numerators and denominators supplied in the publication.

\section{Data analysis}

All data collection/selection, aggregation and analysis activities were carried out using the R statistical programming language [19].

\section{Ethical use of data}

We followed the guidelines from the University of Aberdeen to ensure the ethical use of social media in this study [20]. We also anonymized all the data at the time of collection by removing names, telephone numbers, postcodes, addresses and email addresses. User names were replaced with Study ID numbers. Text from the posts or any other features that might allow for identification of users or source material will never be published or released.

\section{Results}

Results from the study spanning 2004-2015 provided data on 1867 advanced patients, with all providing data on first-line treatment. The most frequently administered first-line monotherapies were sunitinib $(51.9 \%, \mathrm{n}=969)$, pazopanib $(28.0 \%, \mathrm{n}=212)$ and everolimus $(7.5 \%, \mathrm{n}=79)$. For the second line, the most frequently administered monotherapies were sunitinib $(34.0 \%, \mathrm{n}=163)$, pazopanib $(21.2 \%, \mathrm{n}=52)$ and everolimus $(13.2 \%, \mathrm{n}=43)$. For the third line, the most frequently administered monotherapies were sunitinib $(25.0 \%, \mathrm{n}=38)$, everolimus $(19.3 \%, \mathrm{n}=21)$ and pazopanib $(17.0 \%, \mathrm{n}=15)$. For the study spanning 2009-2015, with 1201 advanced patients, the most frequently administered first-line monotherapies were sunitinib $(53.0 \%, \mathrm{n}=637)$, pazopanib $(20.8 \%$, $\mathrm{n}=212)$ and everolimus $(6.8 \%, \mathrm{n}=78)$. For the second line, the most frequently administered monotherapies were sunitinib $(34.7 \%, \mathrm{n}=123)$, pazopanib $(17.0 \%, \mathrm{n}=52)$ and temsirolimus $(13.9 \%, \mathrm{n}=53)$. For the third line, the most frequently administered monotherapies were sunitinib $(22.0 \%, \mathrm{n}=29)$, everolimus $(16.6 \%, \mathrm{n}=21)$ and sorafenib $(14.3 \%, \mathrm{n}=20)$.

The targeted literature search resulted in 24 potentially relevant published studies for patients with metastatic RCC. On applying the search exclusion criteria, five studies were not focused on targeted therapies, ten studies did not contain at least six of the seven targeted therapies of interest, six studies included fewer than 200 subjects, four studies were published prior to 2003 and five studies did not cover the US or EU5 locations (the exclusions listed are not mutually exclusive). Six eligible studies remained for the 2004-2015 comparison and four studies were eligible for the 2009-2015 comparison. The final studies included in the comparisons are detailed in Table 1.

A comparison of the results obtained for the study period of 2004-2015 (Table 2) showed that percentage estimates for most of the targeted therapies were within the variation found across published retrospective studies, with the exception of first-line pazopanib (28.0\%) and first-line axitinib (4.4\%). For the 2009-2015 comparison (Table 3), first-line axitinib (3.3\%) and second-line sunitinib (34.7\%) were higher than the variation in published 
Table 1. Details of published studies included in the comparison.

\begin{tabular}{|llllr|}
\hline Study (year) & Region & Sample size & Study period & Ref. \\
\hline Copher et al. (2016) & USA & 929 & $2009-2015$ & [21] \\
\hline Geynisman et al. (2015) & USA & 1527 & $2004-2010$ & [22] \\
\hline Maroun et al. (2018) & France & 1331 & $2005-2015$ & [23] \\
\hline Miller et al. (2016) & USA & 649 & $2007-2013$ & [24] \\
\hline Jonasch et al. (2014) & USA & 433 & $2010-2012$ & [25] \\
\hline Wagstaff et al. (2015) & UK & 514 & $2009-2012$ & [26] \\
\hline
\end{tabular}

studies, while second-line everolimus (11.9\%) was lower. The remaining 37 comparison points fell within the variation of published studies.

Across the four websites used for the social media study, an examination of the users' countries of origin is shown in Table 4. The USA and UK were the predominant English-speaking countries among the users.

\section{Discussion}

To our knowledge, this work represents one of the first attempts to extract treatment patterns for targeted therapies from health-related social media forum text data in an automated manner. We developed a novel methodological approach using natural language processing, machine learning and rule-based methods. For a valid comparison, it should be noted that the variation in estimates between published studies was considerable (Tables $3 \& 4$ ). This variation can be due to a number of factors, such as geographical differences between different study centers, temporal differences between studies or sampling variation. A large variation between studies indicates that small design differences can lead to large differences in estimates. Given this variation, it is unrealistic to compare estimates from social media with any single study. Therefore, social media-derived estimates were compared with the variation across all selected studies and included the CI estimates for the published studies. It is necessary to include the CIs of the published studies, as they reflect the uncertainty in the estimates based on the sample sizes of published studies.

The proportion estimates obtained for social media fell within the variation of published studies in 19 of 21 cases for the 2004-2015 comparison, and in 18 of 21 cases for the 2009-2015 comparison. For both comparisons, axitinib appeared higher than published estimates for first-line treatment; however, most of the selected studies did not find any use of axitinib as a first-line targeted therapy. The European Society for Medical Oncology [27] and National Institute for Health and Care Excellence [28] guidelines do not recommend axitinib as a first-line treatment, although the National Comprehensive Cancer Network guidelines [29] do include axitinib as a first-line option. There are a number of factors that could be responsible for the differences in social media-derived estimates, including the possibility that more people in younger age groups use social media than people in older age groups, the fact that social media includes clinical trial usage, the treatment options available within current guidelines or the changing treatment landscape. For a new data source such as social media, there is the question of potential bias; however, it is important to remember that no study is free from any bias. The results of this analysis show that social media is a potentially useful and unique approach for assessing treatment patterns, although further research, development and validation of the approach are needed.

Studies based on social media forums have potentially important advantages. They can be carried out relatively quickly due to the algorithmic nature of the methods, and they cost less than other study types. Social media studies can also avoid recruitment problems, and offer insights into conditions where no other data source are available. Posting histories often stretch back more than a decade, which captures changes both historically and in real time. At last, the perspective obtained from social media is an absolute reflection of patient experience and not restricted, limited or framed by responses to questions in a patient interview-type study.

There are also limitations to social media-based studies. Self-reported data may be subject to recall bias, or patients may report treatment information inaccurately due to misunderstanding clinical terms and/or concepts. For example, researchers investigating combination therapies would miss data if patients receiving combination therapy referred to only one of the two treatments by name in their posts. Users of these social media forums may active in more than one forum, leading to some duplication of data. There is also a general perception that older people tend to engage in online activities less frequently than younger people; this may be a potential source of age bias. Caregivers and family members frequently engage online on behalf of patients who cannot, which could mitigate 


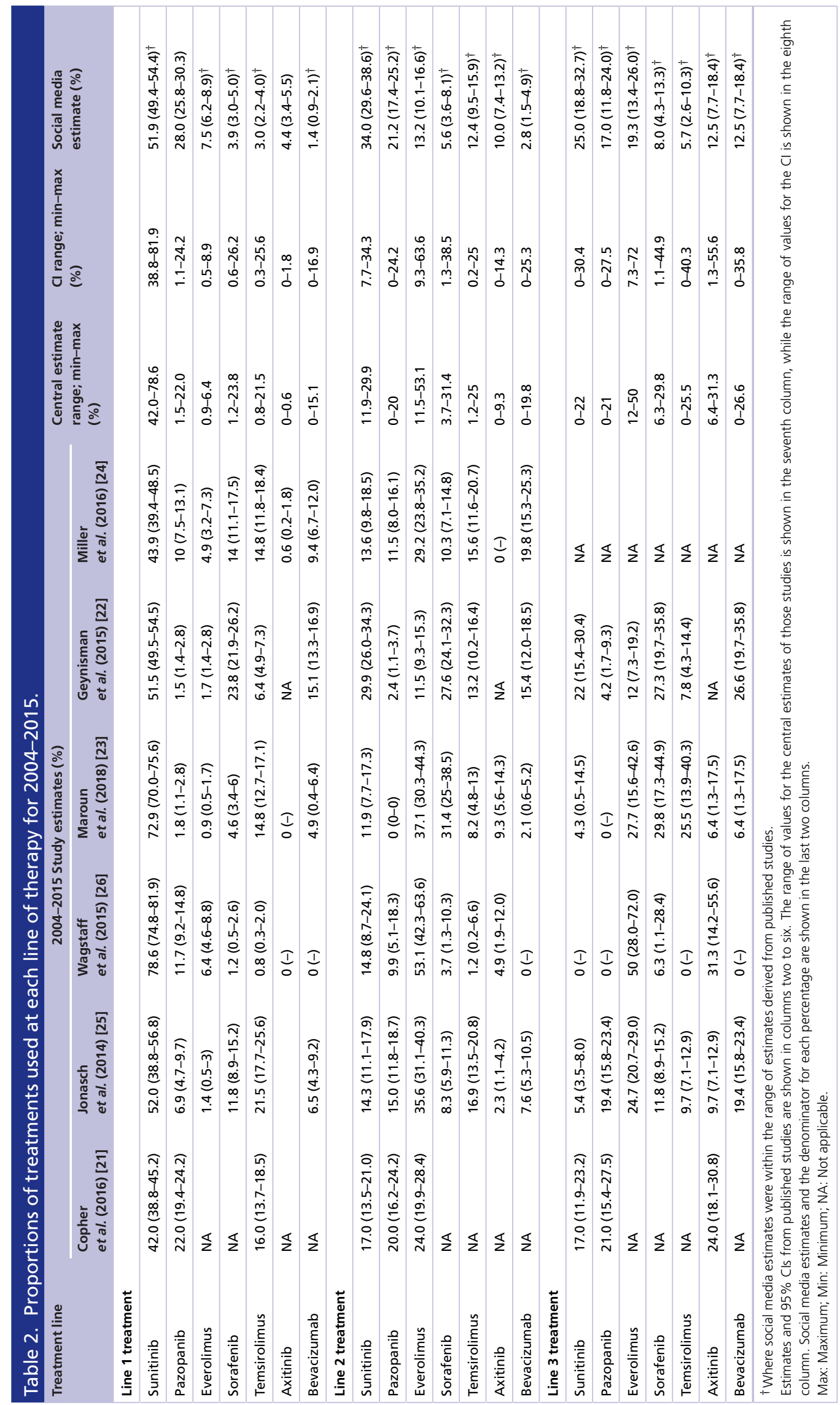


Research Article Ramagopalan, Malcolm, Merinopoulou, McDonald \& Cox

Table 3. Proportions of treatments used at each line of therapy for 2009-2015.

\begin{tabular}{|c|c|c|c|c|c|c|c|}
\hline \multirow[t]{2}{*}{ Treatment line } & \multicolumn{4}{|c|}{ 2009-2015 Study estimates (\%) } & \multirow{2}{*}{$\begin{array}{l}\text { Central estimate } \\
\text { range; min-max } \\
(\%)\end{array}$} & \multirow{2}{*}{$\begin{array}{l}\mathrm{Cl} \text { range; min-max } \\
(\%)\end{array}$} & \multirow{2}{*}{$\begin{array}{l}\text { Social media } \\
\text { estimate (\%) }\end{array}$} \\
\hline & $\begin{array}{l}\text { Copher } \\
\text { et al. (2016) [21] }\end{array}$ & $\begin{array}{l}\text { Jonasch } \\
\text { et al. (2014) [25] }\end{array}$ & $\begin{array}{l}\text { Wagstaff } \\
\text { et al. (2015) [26] }\end{array}$ & $\begin{array}{l}\text { Maroun } \\
\text { et al. (2018) [23] }\end{array}$ & & & \\
\hline \multicolumn{8}{|c|}{ Line 1 treatment } \\
\hline Pazopanib & $22.0(19.4-24.2)$ & $6.9(4.7-9.7)$ & $11.7(9.2-14.8)$ & $1.8(1.1-2.8)$ & $1.8-22.0$ & $1.1-24.2$ & $20.8(18.5-23.3)^{\dagger}$ \\
\hline Everolimus & NA & $1.4(0.5-3)$ & $6.4(4.6-8.8)$ & $0.9(0.5-1.7)$ & $0.9-6.4$ & $0.5-8.8$ & $6.8(5.4-8.4)^{\dagger}$ \\
\hline Axitinib & NA & NA & $0(-)$ & $0(-)$ & 0 & 0 & $3.3(2.4-4.6)$ \\
\hline Bevacizumab & NA & $6.5(4.3-9.2)$ & $0(-)$ & $4.9(0.4-6.4)$ & $0-6.5$ & $0-9.2$ & $2.0(1.3-3.0)^{\dagger}$ \\
\hline \multicolumn{8}{|c|}{ Line 2 treatment } \\
\hline Sunitinib & $17.0(13.5-21.0)$ & $14.3(11.1-17.9)$ & $14.8(8.7-24.1)$ & $11.9(7.7-17.3)$ & $11.9-17.0$ & $7.7-21.0$ & $34.7(29.7-40.0)$ \\
\hline Pazopanib & $20.0(16.2-24.2)$ & $15.0(11.8-18.7)$ & $9.9(5.1-18.3)$ & $0(0-0)$ & $0-20$ & $0-24.2$ & $17.0(13.2-21.5)^{\dagger}$ \\
\hline Axitinib & NA & $2.3(1.1-4.2)$ & $4.9(1.9-12.0)$ & $9.3(5.6-14.3)$ & $2.3-9.3$ & $1.1-14.3$ & $7.7(5.1-11.0)^{\dagger}$ \\
\hline Bevacizumab & NA & $7.6(5.3-10.5)$ & $0(-)$ & $2.1(0.6-5.2)$ & $0-7.6$ & $0-10.5$ & $3.2(1.6-5.7)^{\dagger}$ \\
\hline \multicolumn{8}{|c|}{ Line 3 treatment } \\
\hline Sunitinib & $17.0(11.9-23.2)$ & $5.4(3.5-8.0)$ & $0(-)$ & $4.3(0.5-14.5)$ & $0-17.0$ & $0-23.2$ & $22.0(14.9-29.8)^{\dagger}$ \\
\hline Pazopanib & $21.0(15.4-27.5)$ & $19.4(15.8-23.4)$ & $0(-)$ & $0(-)$ & $0-21$ & $0-27.5$ & $11.8(6.7-18.5)^{\dagger}$ \\
\hline Everolimus & NA & $24.7(20.7-29.0)$ & $50.0(28.0-72.0)$ & $27.7(15.6-42.6)$ & $24.7-50.0$ & $15.6-72.0$ & $16.6(10.4-23.8)^{\dagger}$ \\
\hline Sorafenib & NA & $11.8(8.9-15.2)$ & $6.3(1.1-28.4)$ & $29.8(17.3-44.9)$ & $6.3-29.8$ & $1.1-44.9$ & $14.3(8.5-21.2)^{\dagger}$ \\
\hline Temsirolimus & NA & $9.7(7.1-12.9)$ & $0(-)$ & $25.5(13.9-40.3)$ & $0-25.5$ & $0-40.3$ & $12.1(7.3-19.4)^{\dagger}$ \\
\hline Axitinib & $24.0(18.1-30.8)$ & $9.7(7.1-12.9)$ & $31.3(14.2-55.6)$ & $6.4(1.3-17.5)$ & $6.4-31.3$ & $1.3-55.6$ & $10.0(5.5-16.6)^{\dagger}$ \\
\hline Bevacizumab & NA & $19.4(15.8-23.4)$ & $0(-)$ & $6.4(1.3-17.5)$ & 0-19.4 & $0-23.4$ & $11.3(6.7-18.5)^{\dagger}$ \\
\hline
\end{tabular}

Table 4. Percentages of users from different English-speaking countries from the four web forums used in the social media study.

\begin{tabular}{|lll|}
\hline Forum & Country & Visitors (\%) \\
\hline Cancer survivors network & USA & $48.2 \%$ \\
\hline MacMillan cancer support & UK & $2.9 \%$ \\
\hline & UK & $60.4 \%$ \\
\hline & USA & $16.1 \%$ \\
\hline & Australia & $2.1 \%$ \\
\hline Cancer compass & Canada & $1.4 \%$ \\
\hline & USA & \\
\hline & Canada & $54.0 \%$ \\
\hline Daily strength & & $3.4 \%$ \\
\hline & USA & $29.6 \%$ \\
\hline UK & $3.2 \%$ \\
\hline Canada & $3.0 \%$ \\
\hline
\end{tabular}




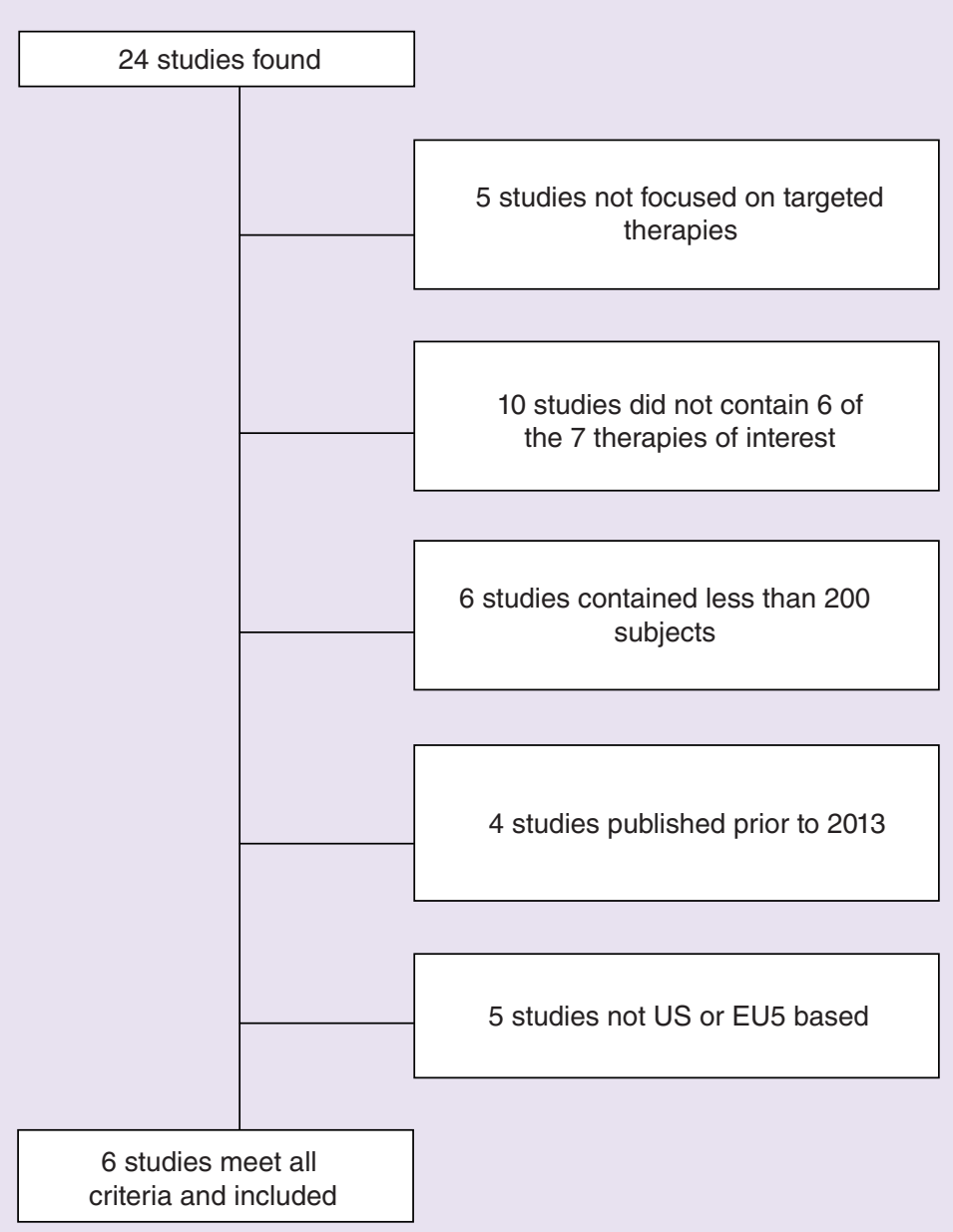

Figure 1. Results of literature review and details of exclusions. Studies may meet multiple exclusion criteria; hence, the number of excluded studies and the included studies do not sum to the 24 found studies.

EU5: France, Germany, Italy, Spain and the UK.

that age bias. The geographical locations of patients involved in social media studies can be difficult to determine, as people can access websites based in other countries. To address this, we assessed the country of origin based on the IP addresses using the sites (Table 4); as the predominant usage was from the USA and Europe, we attempted to select both US and European published studies for a more balanced comparison.

Although there have been advances in machine learning and natural language processing [30], the challenges of this project should not be underestimated. Developing a computer algorithm to do this at scale is a difficult task, and such an approach requires the recognition of subtle linguistic features, such as voice, tense, negation, questions, conditional statements, hypothetical statements and whether the writer is discussing their own treatment history or referring to someone else's. This can be further complicated because, as noted earlier, a high number of users are not patients themselves, but rather caregivers or family members acting on behalf of patients. In addition, the lexical diversity and quality of text in the forums are highly variable, with frequent misspellings, typos, abbreviations and colloquial language.

The exploratory work conducted here shows that there are potential advantages to using social media to determine treatment patterns. This approach can be improved by further developing machine learning algorithms that can detect the relevant sections of text and by better incorporating semantics and parts of speech in tagging information. We can further improve this methodology by using this study to better understand how patients discuss their conditions and treatments. 


\section{Conclusion}

Health-related social media represents an emerging source of data that has potential advantages and could become a tool to provide evidence regarding patient experience. This pilot work demonstrated that estimating treatment patterns from social media is possible, and that the treatment proportions obtained were largely within the variation of estimates from comparable published observational studies. However, variation in published literature was significant and thus further validation should be performed. Areas for improvement have been identified to help to improve the reliability of results obtained.

\section{Future perspective}

Extracting treatment patterns from social media postings using natural language processing has rarely been used. While this approach will likely never replace more traditional methods of identifying patient treatment patterns, we have shown it to be a possible approach. Over the next 5-10 years, this method could initially see greater improvement in accuracy and ease of use, as others develop and improve on the approach. As these improvements are adopted, we would expect to see increasing use of this approach to determine treatment patterns for conditions where data is scarce, hard to access or nonexistent.

\section{Summary points}

- The number of patients turning to health-related social media to share their experiences with other patients has increased over recent years and continues to rise. These discussions contain rich information on patients treatment history, experiences and perceptions.

- This exploratory research was one of the first attempts to extract treatment patterns for targeted therapies from health-related social media forum text data in an automated manner.

- This research uses a novel methodology that combines natural language processing, machine learning and rule-based methods to utilize health-related social media data.

- Comparisons of findings from social media data and estimates from published studies with regard to treatment patterns in renal cell carcinoma show that in 18 of 21 cases for the 2009-2015 period, proportion estimates for social media are within the Cls of published studies.

- Social media studies can be carried out more time-and-cost effectively than other traditional study designs.

- Perspectives obtained from social media are an absolute reflection of patient experience and are not influenced by study setting and/or the presence of a researcher or healthcare professional.

- Limitations to social media-based studies include the potential biases associated with using patient-reported medical histories and noise within the data.

- The research shows that describing treatment patterns using health-related social media data is a possible method of obtaining estimates of treatment patterns where reliable data may not be otherwise available or hard to obtain.

\footnotetext{
Author contributions

SV Ramagopalan, B Malcom, E Merinopoulou, L McDonald and A Cox provided substantial contributions to the conception and design of work, interpretation of results, drafting and revising the work, final approval of the version to be published, and are accountable for all aspects of the work.

Financial \& competing interests disclosure

Funding for this research was provided by Bristol-Myers-Squibb. SV Ramagopalan, B Malcolm and L McDonald are employees of BMS. E Merinopoulou is an employee of Evidera Inc. A Cox was an employee of Evidera Inc. at the time of the study conduct and development of this manuscript. The authors have no other relevant affiliations or financial involvement with any organization or entity with a financial interest in or financial conflict with the subject matter or materials discussed in the manuscript apart from those disclosed.
}

No writing assistance was utilized in the production of this manuscript. 


\section{References}

Papers of special note have been highlighted as: • of interest; $\bullet \bullet$ of considerable interest

1. Price Water House Coopers (PwC). Social media "likes" healthcare: from marketing to social

business (2015). www.pwc.com/us/en/industries/health-industries/library/health-care-social-media.html

2. National Voices and Nesta. Peer Support: what is it and does it work? Summarising evidence from more than 1000 studies (2015). www.nationalvoices.org.uk/sites/default/files/public/publications/peer_support_-_what_is_it_and_does_it_work.pdf

3. Brennan S. Why I turned to social media for help after a double lung transplant: online health forums are increasingly used as a way of getting peer support and taking control of an illness. The

Guardian (2016). www.theguardian.com/society/2016/mar/01/social-media-control-illness-online-health-forums

4. Pendry L, Salvatore J. Individual and social benefits of online discussion forums. Comp. Hum. Behav. 50, 211-220 (2015).

* This study assessed the benefits of engaging in social media discussion forums for users' well-being. The findings suggested that these forums can have a benefit at both individual and societal levels, and are also linked to well-being for stigmatised groups, their satisfaction in life and civic offline engagements.It partly helps explain the increasing use of social media forums by patients or caregivers that we observe in recent years.

5. Ennis-O'Connor M. How online patient communities are changing the face of cancer care (2014). https://blogs.bmj.com/ebn/2014/03/03/how-online-patient-communities-are-changing-the-face-of-cancer-care/

6. Allen C, Vassilev I, Kennedy A, Rogers A. Long-term condition self-management support in online communities: a meta-synthesis of qualitative papers. J. Med. Internet Res.18(3), e61 (2016).

7. American Cancer Society. Cancer survivors network. https://csn.cancer.org/

8. Diabetes Daily. Join the Diabetes Forum - Don't Just Live, Thrive! www.diabetesdaily.com/

9. Medwatcher Mobile Application. MedWatcher Enterprise. www.fda.gov/medical-devices/medical-device-reporting-mdr-how-report-me dical-device-problems/medwatcher-mobile-app

10. US Food and Drug Administration. Patient-focused drug development: collecting comprehensive and representative input guidance for industry, Food and Drug Administration staff, and other stakeholders: draft guidance (2018). www.fda.gov/media/113653/download

** The guidance from the US Food and Drug Administration includedthe use of patient reported data from social media forums as an additional wayto incorporate the patient perspective and subsequently inform traditionalmethods of research. This guidance demonstrates a wide acknowledgement of theimportance of this new type of data source in healthcare research.

11. CancerCompass. CancerCompass message board (2019). www.cancercompass.com/message-board.htm

12. DailyStrength. Getting better together (2019). www.dailystrength.org/

13. Macmillan Cancer Support. Online community (2019). www.macmillan.org.uk/

14. Alexa, an Amazon Company. Find, reach, and convert your audience with marketing that works. www.alexa.com/

15. Unicode Inc. The Unicode ${ }^{\circledR}$ standard version 11.0 - core specification (2018). https://unicode.org/versions/Unicode11.0.0/

16. Github Inc. High-performance stemmer, tokenizer, and spell checker for R. https://github.com/ropensci/hunspell

17. Levenshtein V. Binary codes capable of correcting deletions, insertions, and reversals. Soviet Physics Doklady 10(8), 707-710 (1966).

18. Savova GK, Masanz JJ, Ogren PV et al. Mayo clinical Text Analysis and Knowledge Extraction System (cTAKES): architecture, component evaluation and applications. J. Am. Med. Inform. Assoc.17(5), 507-513 (2010).

19. R Core Team R. A language and environment for statistical computing. R Foundation for Statistical Computing, Vienna, Austria (2017). www.R-project.org

20. Townsend L, Wallace C. Social media research: a guide to ethics. www.gla.ac.uk/media/media_487729_en.pdf

*Social media dataprovide huge opportunity to gather data that it would be otherwise difficult orcostly to obtain. Although traditional ethics frameworks can inform researchersto some extent, social media data are new and unique and therefore bring new challengesin ensuring ethical research. These guidelines were developed to ensure that howwe obtain and reuse such data is done to the highest possible ethicalstandards.

21. Copher R, Dacosta Byfield S, Buzinec P, Korrer S, Baig M Understanding real worldtreatment patterns, healthcare resource utilization (HRU) and costs amongmetastatic renal cell carcinoma (mRCC) patients. Ann. Oncol. 27(Suppl. 6), vi351-vi358 (2016).

22. Geynisman DM, Hu JC, Liu L et al. Treatment patterns andcosts for metastatic renal cell carcinoma patients with private insurance inthe United States. Clin. Genitourin. Cancer 13(2), e93-e100 (2015).

23. Maroun R, Mitrofan L, Benjamin L et al. Real life patterns of careand progression free survival in metastatic renal cell carcinoma patients:retrospective analysis of cross-sectional data. BMC Cancer 18(1), 214 (2018).

24. Miller LA, Stemkowski S, Saverno K et al. Patterns of care inpatients with metastatic renal cell carcinoma among a U.S. payer populationwith commercial or Medicare Advantage membership. J. Manag. Care Spec. Pharm. 22(3), 219-226 (2016).

25. Jonasch E, Signorovitch JE, Lin PL et al. Treatment patterns inmetastatic renal cell carcinoma: a retrospective review of medical records from US community oncology practices. Curr. Med. Res. Opin. 30(10), 2041-2050 (2014). 
26. Wagstaff J, Jones R, Hawkins $\mathrm{R}$ et al. Treatment patterns andclinical outcomes in patients with renal cell carcinoma in the UK: insightsfrom the RECCORD registry. Ann. Oncol. 27(1), 159-165 (2015).

27. Escudier B, Porta C, Schmidinger M et al. Renal cell carcinoma: ESMO Clinical Practice Guidelines for diagnosis, treatment and follow-up. Ann. Oncol.27(Suppl. 5), v58-v68 (2016).

28. National Institute for Health and Care Excellence.Renal cancer overview - interactive flow chart (2019). https://pathways.nice.org.uk/pathways/renal-cancer

29. Motzer RJ, Jonasch E, Agarwal N et al. Kidney Cancer, Version 2.2017, NCCN Clinical Practice Guidelines in Oncology. J. Natl Compr. Canc. Netw.15(6), 804-834 (2017).

30. Hirschberg J, Manning CD. Advances in natural language processing. Science 349(6245), 261-266 (2015).

*This publication discusses advances in natural language processing in recent years that highlight how rapidly this area is evolving, and therefore enabling researchers to harvest and analyse data that previously would not have been possible - such as the unstructured data from social media forums that were used in our study. 